\title{
Growth of GaAsBi/GaAs Multi Quantum Wells on (100) GaAs Substrates by Molecular Beam Epitaxy*
}

\author{
P. Patil, ${ }^{\dagger}$ T. Tatebe, Y. Nabara, K. Higaki, N. Nishii, S. Tanaka, F. Ishikawa, and S. Shimomura \\ Graduate School of Science and Engineering, Ehime University, \\ 3 Bunkyo-cho, Matsuyama, Ehime 790-8577, Japan
}

(Received 15 January 2015; Accepted 16 November 2015; Published 12 December 2015)

\begin{abstract}
Multi-quantum wells (MQWs) consisting of 3 or 3.5 pairs of nominally 8.8-nm-thick GaAs layers and 5.6-nmthick $\mathrm{GaAs}_{0.97} \mathrm{Bi}_{0.03}$ were grown by molecular beam epitaxy with varying the growth temperature of GaAs layers, $T_{\mathrm{GaAs}}$, and keeping the growth temperature of the $\mathrm{GaAsBi}$ layers at $350^{\circ} \mathrm{C}$ and their $\mathrm{Bi}$ compositional structure and optical properties were investigated. Analysis of x-ray diffraction spectra reveals that $67 \%$ of the total Bi atoms supplied during growth of a single GaAsBi layer were segregated on the growing surface and were incorporated into the successive GaAs layer at $T_{\mathrm{GaAs}}=350^{\circ} \mathrm{C}$. The GaAs layers at $T_{\mathrm{GaAs}}=450$ and $500^{\circ} \mathrm{C}$ contained $17 \%$ of the Bi atoms totally supplied and $50 \%$ of them were evaporated. Almost all Bi atoms segregated during growth of GaAsBi evaporated and were not incorporated into the GaAs layer at $T_{\mathrm{GaAs}}=550^{\circ} \mathrm{C}$ or higher. Photoluminescence $(\mathrm{PL})$ spectra at $13 \mathrm{~K}$ shows all MQW samples have good optical quality and the MQW sample grown at $T_{\mathrm{GaAs}}=550^{\circ} \mathrm{C}$ shows the longest wavelength emission peak at $1116 \mathrm{~nm}$ which is $44 \mathrm{~nm}$ longer than the PL wavelength for the MQW grown at $T_{\mathrm{GaAs}}=350^{\circ} \mathrm{C}$, even though the tremendous reduction of Bi incorporation into the GaAs layer grown at $T_{\mathrm{GaAs}}=550^{\circ} \mathrm{C}$. The result strongly suggests that $\mathrm{GaAsBi} / \mathrm{GaAs}$ has the type II band configuration. [DOI: $10.1380 /$ ejssnt.2015.469]
\end{abstract}

Keywords: GaAsBi; Multi-quantum wells; Molecular beam epitaxy; X-ray diffraction; Photoluminescence

\section{INTRODUCTION}

The development of new semiconductor heterostructures with GaAsBi has attracted much scientific interest recently as it has potential applications such as lasers [1, 2], transistors [3], photo-detectors [4], spintronic devices [5], solar cells [6], because the energy gap of $\mathrm{GaAsBi}$ is much narrower than those of InGaAs, GaAsSb and various III-V compounds with the same strain due to lattice mismatch to GaAs. The band gap energy of GaAsBi alloy pseudomorphically grown on a $\operatorname{GaAs}(100)$ substrate is reduced at a rate of $86 \mathrm{meV} / \%$ with increasing the $\mathrm{Bi}$ composition in \% [7], and also shows the temperature insensibility $[8,9]$. Increase of the valence band maxima of GaAsBi arises from an anti-crossing interaction between the valence band of GaAs and $\mathrm{Bi}$ resonant states which reduces the energy of the conduction band $[10,11]$. The downward movement of the conduction band has also been demonstrated using room temperature photoreflectance spectra, which implies that GaAsBi/GaAs system has type I band configuration [12].

The growth of GaAsBi has proven complicated, requiring very low growth temperatures $\left(<400^{\circ} \mathrm{C}\right)$ to incorporate significant fractions of $\mathrm{Bi}[7,13]$. The quality of GaAsBi is highly dependent on the $\mathrm{Bi}$ composition and the growth temperature. For particular growth condition, the flux also must be accurately controlled as a shortage of Bi may lead to surface roughness while excess $\mathrm{Bi}$ causes formation of $\mathrm{Bi}$ droplet [14]. Bismuth, which has an atomic mass number of 208, is the heaviest nonradioactive element in the periodic table. The $\mathrm{Bi}$ atom is approximately 25\% larger in radius than $\mathrm{Ga}$ and $\mathrm{As}$ atoms, and tends to surface-segregate during growth [15],

\footnotetext{
* This paper was presented at the 7th International Symposium on Surface Science, Shimane Prefectural Convention Center $\mathrm{Ku}$ nibiki Messe), Matsue, Japan, November 2-6, 2014.

$\dagger$ Corresponding author: pals4m@yahoo.in
}

furthermore, improving the electronic properties of the material as a surfactant [16]. Hence, the composition of $\mathrm{Bi}$ in $\mathrm{GaAs}_{1-x} \mathrm{Bi}_{x}$ is strongly dependent on the growth temperature and is determined by a more complicated mechanism than that of conventional III-V alloys, e.g., InGaAs or AlGaAs. In the growth of GaAsBi/GaAs/AlGaAs separate confinement heterostructures, the growth temperature of the top AlGaAs barrier is restricted by the growth of annealing temperature limits of the previous layers [17]. In this study, we grew GaAsBi/GaAs multi quantum wells (MQWs) sandwiched between AlGaAs layers by molecular beam epitaxy and investigated the dependence of x-ray diffraction spectrum and low temperature photoluminescence from MQWs on the growth temperature of GaAs layers with fixing the growth temperature of GaAsBi layers at $350^{\circ} \mathrm{C}$.

\section{EXPERIMENTAL}

The GaAs substrate was etched by semico clean solution (Furuuchi Chemical Corp.) for $1 \mathrm{hr}$, transferred into an VG semicon V80H MK-III MBE system equipped with $\mathrm{Ga}, \mathrm{Al}, \mathrm{As}$ and $\mathrm{Bi}$ conventional K-cells. Beam equivalent pressures (BEPs) of $\mathrm{Ga}, \mathrm{Al}, \mathrm{As}_{4}$ and $\mathrm{Bi}$ were adjusted by an ion gauge as Ga flux $8.5 \times 10^{-7}$ mbar, As4 flux $9.6 \times 10^{-6}$ mbar, Bi flux $5.4 \times 10^{-7}$ mbar and Al flux $1.9 \times 10^{-7}$ mbar. The substrates were baked at a temperature of $400^{\circ} \mathrm{C}$ for $30 \mathrm{~min}$, loaded into a growth chamber, and heated at $650^{\circ} \mathrm{C}$ for $5 \mathrm{~min}$ to remove the native surface oxide. A schematic illustration of the sample structure is shown in the Fig. 1. The samples comprise a $160 \mathrm{~nm}$ GaAs buffer grown at $580^{\circ} \mathrm{C}$, followed by $112-\mathrm{nm}$ AlGaAs layer grown at $640^{\circ} \mathrm{C}, 3$ (3.5) alternating layers of $8.8-\mathrm{nm}$ GaAs at $T_{\mathrm{GaAs}}=350(450,500,550$, and $600)^{\circ} \mathrm{C}$ and $5.6-\mathrm{nm} \mathrm{GaAsBi}$ at the growth temperature of $\mathrm{GaAsBi}, T_{\mathrm{GaAs}}=350^{\circ} \mathrm{C}$, and $112-\mathrm{nm}$ AlGaAs layers were stacked at $640^{\circ} \mathrm{C}$ with a $24-n m$ GaAs cap layer grown at $580^{\circ} \mathrm{C}$. For the growth of the sample with 3.5 alternat- 


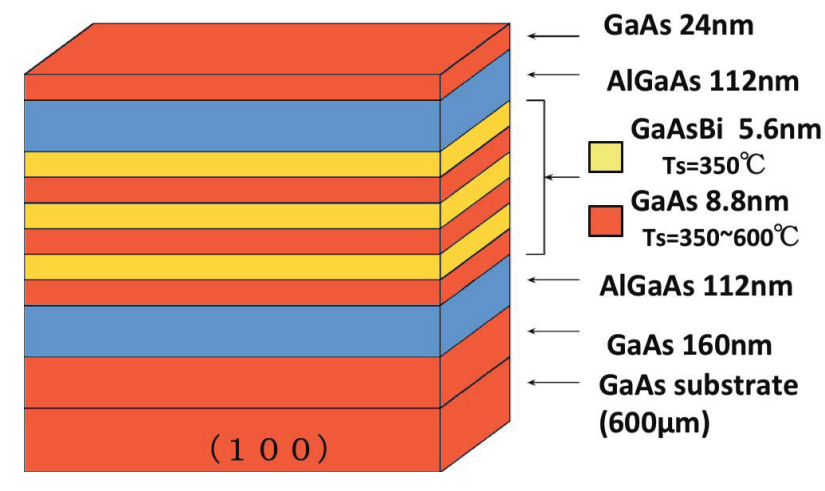

FIG. 1. Schematic structure of GaAsBi/GaAs multi quantum well (MQW) sample.

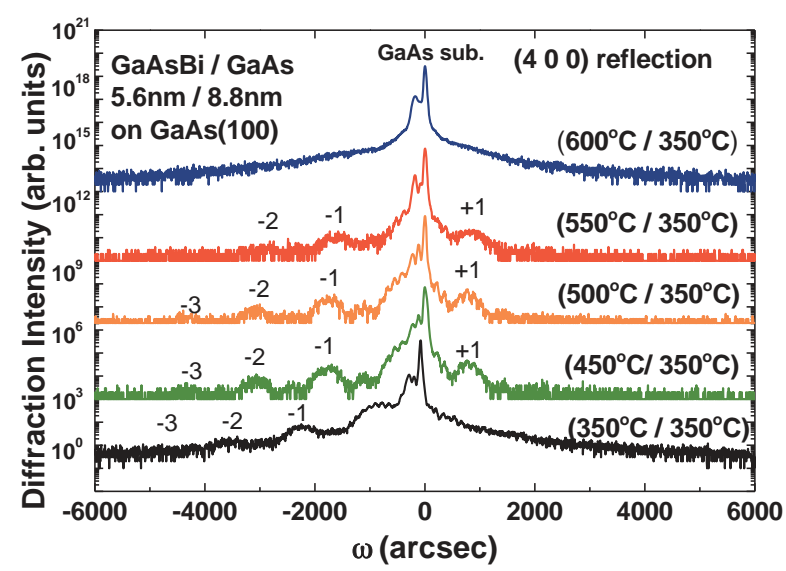

FIG. 2. XRD spectra from (100) GaAs/GaAsBi MQW dependent on growth temperature of GaAs layers.

ing layer pairs, a 8.8-nm GaAs layer was added after the growth of the 3 pairs. The time interval between every epilayer was 5 to 18 min to change and set the substrate temperature accurately for the growth of the following layer.

High-resolution x-ray diffraction (HRXRD) was measured by a Phillips X'Pert Pro Materials Research Diffractometer with an incident x-ray beam wavelength of $\mathrm{CuK}_{\alpha}$, $(\lambda=0.15406 \mathrm{~nm})$. The samples were mounted on a computer controlled goniometer stage. The XRD scans were then fitted using Epitaxy software to determine the $\mathrm{Bi}$ composition and the epilayer thicknesses assuming a $\mathrm{GaBi}$ lattice constant of $6.324 \AA$ [18].

The optical properties of the sample were characterized by low-temperature $(13 \mathrm{~K})$ photoluminescence $(\mathrm{PL})$ spectroscopy. The wavelength of an excitation laser was $442 \mathrm{~nm}$ (He-Cd laser, $10 \mathrm{~mW}$ ) and focused on a sample surface area of about $200 \mu \mathrm{m}$ diameter.

\section{RESULTS AND DISCUSSION}

Figure 2 shows the x-ray diffraction patterns of $\theta$ $2 \theta$ scans from reflection obtained from GaAsBi/GaAs MQWs grown at $T_{\mathrm{GaAs}}=350-600^{\circ} \mathrm{C}$. The sharp and largest peak located at 0 arcsec in each diffraction pattern

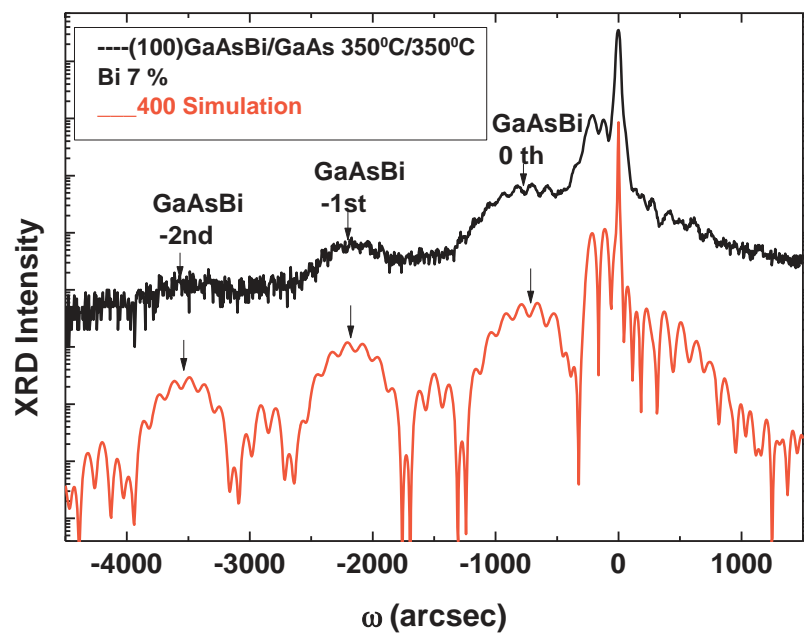

FIG. 3. Measured XRD spectra (black) and simulated spectra (red) for the $\mathrm{GaAs} / \mathrm{GaAsBi}\left(350^{\circ} \mathrm{C} / 350^{\circ} \mathrm{C}\right) \mathrm{MQW}$ sample.

corresponds to the diffraction from the GaAs buffer layer and substrate. The $2^{\text {nd }}$ and $3^{\text {rd }}$ largest peaks near -200 arcsec in diffraction patterns from MQW samples grown at $T_{\mathrm{GaAs}}=350$ to $550^{\circ} \mathrm{C}$ is from the double AlGaAs epilayers and the two peaks are joined into a single peak in the diffraction patterns from MQWs grown at $T_{\mathrm{GaAs}}$ $=600^{\circ} \mathrm{C}$ due to lateral fluctuation of the layer thickness caused by surface and interface roughness. Peaks from the GaAsBi/GaAs alternating layers are labeled by $-3^{\text {rd }}$ to $1^{\text {st }}$, as usually done in x-ray diffraction patterns from a superlattice structures. The 0th peak labeled in the XRD spectra is a main peak and its diffraction angle position is determined by the average Bi composition of overall GaAsBi/GaAs layers. The others are satellite peaks and their angle position spacing is given by the period of the GaAsBi/GaAs layers. A striking difference between diffraction patterns from samples grown at $T_{\mathrm{GaAs}}=350^{\circ} \mathrm{C}$ and $450^{\circ} \mathrm{C}$ is those peak positions and intensity. The $-2^{\text {nd }}$, $-1^{\text {st }}$, and 0th peak are observed at $-3600,-2200,-800$ arcsec in the diffraction pattern from the sample grown at $T_{\mathrm{GaAs}}=350^{\circ} \mathrm{C}$. For the sample at $T_{\mathrm{GaAs}}=450^{\circ} \mathrm{C}$, those peaks are shifted by 500 arcsec to the higher angle side, indicating reduction of the averaged Bi composition [7]. The diffraction pattern from the sample grown at $T_{\mathrm{GaAs}}$ $=500^{\circ} \mathrm{C}$ is almost the same as that from the sample at $T_{\mathrm{GaAs}}=450^{\circ} \mathrm{C}$. For the sample grown at $T_{\mathrm{GaAs}}=550^{\circ} \mathrm{C}$, the satellite peaks are observed at 200 arcsec higher angles compared to the samples at $T_{\mathrm{GaAs}}=450$ and $500^{\circ} \mathrm{C}$. It shows $550^{\circ} \mathrm{C}$ sample has a slightly smaller Bi composition than 450 and $500^{\circ}$ Csamples. Although the sample grown at $T_{\mathrm{GaAs}}=600^{\circ} \mathrm{C}$ shows much smaller satellite peak intensity comparing to other patterns, their positions are almost the same as the sample grown at $T_{\mathrm{GaAs}}=550^{\circ} \mathrm{C}$ indicating the same $\mathrm{Bi}$ composition in the MQW.

Experimental and simulated curves of XRD for the sample grown at $T_{\mathrm{GaAs}}=350^{\circ} \mathrm{C}$ are shown Fig 3 . In the simulation the $\mathrm{Bi}$ composition of the GaAsBi layers is $7 \%$ where it is assumed that all $\mathrm{Bi}$ atoms are uniformly contained in GaAsBi layers and there are no Bi atoms in GaAs layers. The simulated curve well reproduces the 


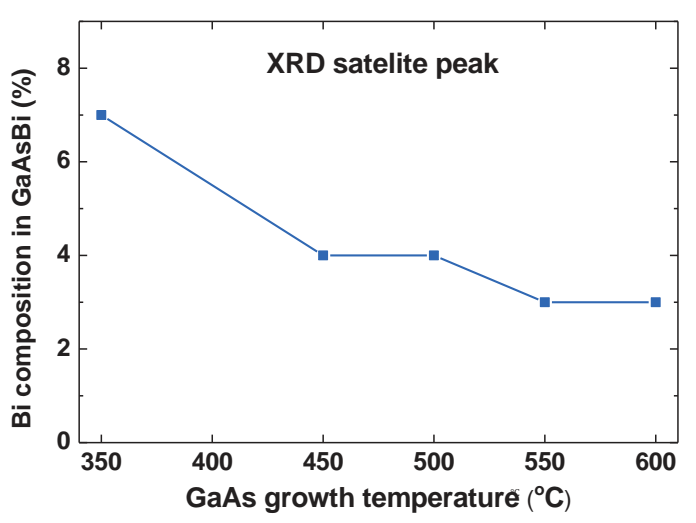

FIG. 4. Bi composition of the GaAsBi layers is plotted in blue square as a function of the growth temperature of GaAs. The Bi Composition was determined by XRD simulation to reproduce the satellite peak position based on a structure where GaAsBi layers have all $\mathrm{Bi}$ atoms and uniform $\mathrm{Bi}$ composition.

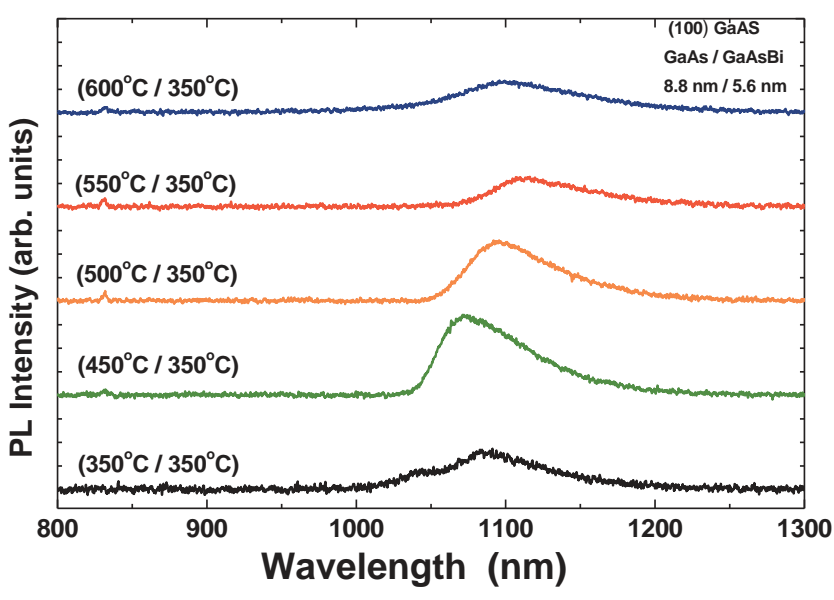

FIG. 5. Photoluminescence spectra from (100) GaAsBi/GaAs MQW samples at $13 \mathrm{~K}$.

diffraction peak position and spacing of the satellite peaks including the 0th peak. Some amount of Bi atoms was segregated on surfaces and incorporated into successive GaAs layers. This reduces the compositional modulation, the intensity ratio of the $-1^{\text {st }}\left(-2^{\text {nd }}\right)$ peak to 0 th peak, whose value is $0.10(0.027)$ for the experimental pattern and $0.19(0.042)$ for the simulated pattern. It is noted that the satellite peak position and intensity do not change by changing the number of pairs of GaAs and GaAsBi layers from 3 to 3.5 .

Deduced values of the $\mathrm{Bi}$ composition of the GaAsBi layers are plotted as a function of $T_{\mathrm{GaAs}}$ in Fig. 4, where it was assumed that GaAsBi layers has all Bi atoms and uniform $\mathrm{Bi}$ composition. The $\mathrm{Bi}$ composition is decreasing dramatically from $7 \%$ to $3 \%$ with increasing the growth temperature of GaAs from 350 to $600{ }^{\circ} \mathrm{C}$. The result shows that $\mathrm{Bi}$ atoms segregate on the surface during growth of a GaAsBi layer. Some of them evaporate in increasing the substrate temperature up to $T_{\mathrm{GaAs}}$, the other atoms are incorporated during growth of the succes- sive GaAs layer. Taking it into account that the Bi cell temperature was $610{ }^{\circ} \mathrm{C}$, all of the surface-segregated $\mathrm{Bi}$ atoms on the surface of the GaAsBi layer evaporate when substrate temperature was increased up to $600{ }^{\circ} \mathrm{C}$. Hence the GaAs layer grown at $600^{\circ} \mathrm{C}$ after the growth of the GaAsBi layer does not contain $\mathrm{Bi}$ or As atoms. The Bi composition obtained our XRD simulation for the sample grown at $T_{\mathrm{GaAs}}=600^{\circ} \mathrm{C}$ is considered to be the actual $\mathrm{Bi}$ composition of the GaAsBi layer and its value is 0.03 . The actual Bi composition of GaAsBi layers is the same for all samples because they were grown at the same temperature and there are almost no Bi atoms on the surface after the growth of GaAs. The Bi composition of the GaAsBi layer from our XRD simulation includes the contribution of incorporation of segregated $\mathrm{Bi}$ atoms into GaAs layers for the sample grown at $350^{\circ} \mathrm{C}$. The vapor pressure of $\mathrm{Bi}$ from the $\mathrm{K}$-cell is a good guide indicator to consider the evaporation of $\mathrm{Bi}$ atoms from the surface. The beam equivalent pressure of $\mathrm{Bi}$ atoms supplied from the $\mathrm{K}$-cell at $350^{\circ} \mathrm{C}$ is $1.0 \times 10^{-10}$ mbar and is smaller by 3 orders of magnitude than that at $610^{\circ} \mathrm{C}\left(5.4 \times 10^{-7} \mathrm{mbar}\right)$. It implies that $67 \%$ of $\mathrm{Bi}$ atoms supplied during growth of the GaAsBi layer at $350^{\circ} \mathrm{C}$ were segregated and were incorporated into the successive GaAs layer grown at $T_{\mathrm{GaAs}}=350{ }^{\circ} \mathrm{C}$. When the sample was grown at $T_{\mathrm{GaAs}}=450$ or $500{ }^{\circ} \mathrm{C}, 50 \%$ of them evaporated and $17 \%$ were incorporated into GaAs. In the analysis, we consider the difference of the structure of samples. The result may suggest that substrate temperature range from 450 to $500{ }^{\circ} \mathrm{C}, \mathrm{Bi}$ atoms bound to $\mathrm{Ga}$ atoms remain on the surface, while the $\mathrm{Bi}$ atoms bound to a Bi or As atoms are desorbed due to small bond strength. The Bi atoms bound to $\mathrm{Ga}$ atoms evaporate higher than $550{ }^{\circ} \mathrm{C}$, all of segregated $\mathrm{Bi}$ atoms cannot remain on the surface. We also should consider interdiffusion of As and $\mathrm{Bi}$ atoms in the analysis. Makholoufi, however, reported that there is no out-diffusion of $\mathrm{Bi}$ atoms from GaAsBi layer to the GaAs layer up to $650^{\circ} \mathrm{C}$ [19].

Low temperature $(13 \mathrm{~K}) \mathrm{PL}$ spectrum from each sample is shown in Fig. 5. The main peak of the PL spectra from the samples was observed from $1069 \mathrm{~nm}$ to $1116 \mathrm{~nm}$ and their full width at half maximum (FWHM) was 70 meV except for the sample $T_{\mathrm{GaAs}}=600{ }^{\circ} \mathrm{C}(\mathrm{FWHM}=$ $90 \mathrm{meV})$ [7]. The GaAsBi/GaAs MQW sample grown at $T_{\mathrm{GaAs}}=550^{\circ} \mathrm{C}$ has the longest $\mathrm{PL}$ peak wavelength and its value is $\lambda=1116 \mathrm{~nm}$ (the value of photon energy is $h \nu=1.111 \mathrm{eV})$. Even though the tremendous reduction of $\mathrm{Bi}$ incorporation into GaAs layers was observed due to increase of $T_{\mathrm{GaAs}}$, small change of the emission wavelength or red shift of the PL emission implies that the GaAsBi/GaAs MQW has the type II band configuration. The luminescence is generated by the recombination of the electrons and holes accumulated in different layers due to wavefunction penetration. If the $\mathrm{GaAsBi} / \mathrm{GaAs}$ MQW has the type I band, reduction of $\mathrm{Bi}$ incorporation into the GaAs layer causes large blue shift in PL spectra.

Figure 6 shows a schematic illustration of band configuration of both samples grown at $T_{\mathrm{GaAs}}=350^{\circ} \mathrm{C}$ and at $T_{\mathrm{GaAs}}=550{ }^{\circ} \mathrm{C}$. In the figure, we omit the GaAs layer between the GaAsBi layer and the AlGaAs layer for the sample grown at $T_{\mathrm{GaAs}}=550^{\circ} \mathrm{C}$ for the simplicity. A large valence band offset and small and negative conduction band offset are expected because a Bi atom has higher s-orbital and p-orbital energies than an As atom. Holes 

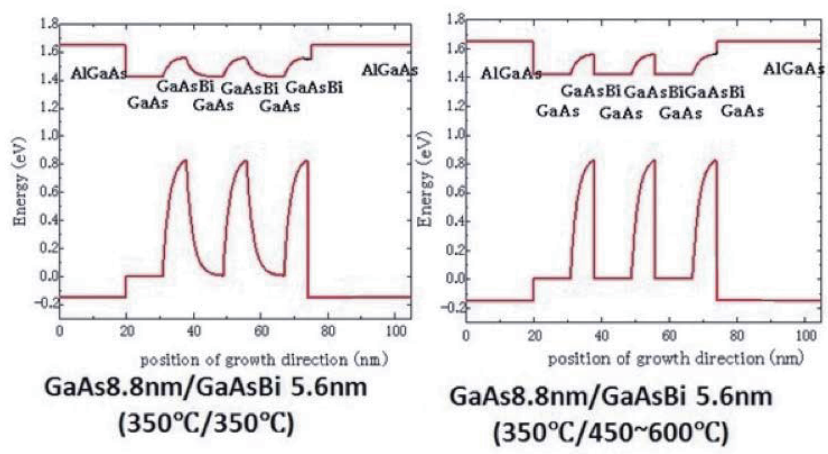

FIG. 6. Band configuration for the GaAsBi/GaAs MQW samples grown at $T_{\mathrm{GaAs}}=350^{\circ} \mathrm{C}$ and $550^{\circ} \mathrm{C}$.

are confined in the GaAsBi layer, and electrons are in the GaAs layer. For the sample grown at $T_{\mathrm{GaAs}}=350{ }^{\circ} \mathrm{C}$, the $1^{\text {st }}$ GaAs layer which is located in the substrate side does not have $\mathrm{Bi}$ atoms. On the other hand, $2^{\text {nd }}$ and $3^{\text {rd }}$ GaAs layers were grown after the growth of the $1^{\text {st }}$ and $2^{\text {nd }}$ GaAsBi layers, respectively. Therefore segregated Bi atoms are incorporated into $2^{\text {nd }}$ and $3^{\text {rd }}$ GaAs layers. The incorporation of Bi changes the bandgap energy and raises the energy level of conduction electron in the $2^{\text {nd }}$ and $3^{\text {rd }}$ GaAs layers. Origin of the two peaks observed in the PL spectrum from the sample grown at $T_{\mathrm{GaAs}}=350^{\circ} \mathrm{C}$ in Fig. 5 is reasonably explained. The main peak comes from the transition between the electron states in the $1^{\text {st }} \mathrm{GaAs}$ layer to the hole states in the $1^{\text {st }}$ GaAsBi layer. The small peak observed in the short wavelength side of the main PL peak corresponds to the transition from the ground state of electrons in the $2^{\text {nd }}$ and $3^{\text {rd }}$ GaAs layers to the ground state of holes in the GaAsBi layers. Small intensity of the shorter wavelength peak is due to the reduction of overlap between wavefunctions of the electrons and holes. On the other hand, the three GaAs layers of the sample grown at $T_{\mathrm{GaAs}}=550^{\circ} \mathrm{C}$ do not contain $\mathrm{Bi}$ atoms and $\mathrm{PL}$ shows the single peak. This result indicates that we have realized GaAsBi/GaAs MQW without Bi incorporation into GaAs layers and with good optical quality. The band configuration of GaAsBi/GaAs is highly controversial. Tight binding band calculation of GaAsBi based on photoreflectance result suggests that GaAsBi/GaAs has the type I band configuration $[20,21]$. Very short decay time (70 ps) obtained by time resolved PL for a single 7.5-nm-thick GaAsBi QW at RT implies that it may have the type I band configuration. In contrast to type-I semiconductor structures, the energy minima for electrons and holes lie in different layers in type II semiconductor structure. The defining feature of the type-II quantum well is the spatial separation of electron and hole confinement in the epitaxial growth direction at low temperatures. To determine the type of band configuration by PL measurement, photoexcited carrier densities of electrons and holes must be enough small to keep the flat band and also a sample must be at low temperature not to detect photons from the inner layer transition between thermally excited electron and holes. Actually the type II to type I transition was observed in GaAs/GaAsSb system with increasing photoexcitation power $[22,23]$. On the other hand, there are reports which interpret that luminescence from a single GaAsBi/GaAs QW consists of lower energy photons due to localized electron hole pairs and higher energy photons from the band edge transition [24, 25]. For the evaluation of the band configuration of $\mathrm{GaAsBi} / \mathrm{GaAs}$, we prepared GaAsBi/GaAs MQW consisting of less than $10 \mathrm{~nm}$ thick layers and abrupt interface where electrostatic potential produced by charge separation and accumulation is not large and spatially separated electron and holes are recombined using the overlap of their wavefunctions which penetrate through interface and barrier potential. The GaAsBi/GaAs MQW grown by Authors has a suitable structure for determining band configuration and measurement of PL was carried out at low temperature (13 $\mathrm{K})$ under a low power excitation (10 mW, $442 \mathrm{~nm})$.

\section{CONCLUSIONS}

Molecular beam epitaxial growth of $\mathrm{GaAs}_{0.97} \mathrm{Bi}_{0.03} / \mathrm{GaAs}(5.6 \mathrm{~nm} / 8.8 \mathrm{~nm}) \mathrm{MQW}$ on a (100) GaAs substrate with changing the growth temperature of the GaAs layer from $T_{\mathrm{GaAs}}=350$ to $600^{\circ} \mathrm{C}$ was investigated. The $\mathrm{Bi}$ atoms remain on the growing surface during growth of both GaAsBi and GaAs layers at a low temperature growth of $350^{\circ} \mathrm{C}$. Analysis of the XRD spectrum of each sample revealed not only the amount of $\mathrm{Bi}$ atoms segregated during growth of GaAsBi at $350^{\circ} \mathrm{C}$ and also the amount of $\mathrm{Bi}$ atoms evaporated with increasing the substrate temperature prior to the growth of GaAs layer. We have realized the GaAsBi/GaAs MQW without incorporation of Bi into the GaAs layers and with good optical quality. It was confirmed that GaAsBi/GaAs has the type II band configuration.

\section{ACKNOWLEDGMENTS}

The authors gratefully acknowledge the Ministry of social justice and welfare, India for Doctoral Fellowship (P. Patil, No. DSW/Edu/2012-2013/D-IV/564).
[1] Y. Tominaga, K. Oe, and M. Yoshimoto, Appl. Phys. Express 3, 062201 (2010).

[2] P. Ludewig, N. Knaub, N. Husain, S. Reinhard, L. Nattermann, I. P. Marko, S. R. Jin, K. Hild, S. Chatterjee, W. Stolz, S. J. Sweeney, and K. Volz, Appl. Phys. Lett. 102, 242115 (2013).
[3] Z. D. Marks, I. W. Haygood, and B. Van Zeghbroeck, IEEE Trans. Electron Devices 60, 200 (2013).

[4] C. J. Hunter, F. Bastiman, A. R. Mohmad, R. Richards, J. S. Ng, S. J. Sweeney, and J. P. R. David, IEEE Photon. Tech. Lett. 24, 2191 (2012).

[5] B. Fluegel, S. Francoeur, A. Mascarenhas, S. Tixier, E. C. Young, and T. Tiedje, Phys. Rev. Lett. 97, 067205 
(2006).

[6] R. N. Kini, L. Bhusal, A. J. Ptak, R. France, and A. Mascarenhas, J. Appl. Phys. 106, 043705 (2009).

[7] S. Tixier, M. Adamcyk, T. Tiedje, S. Francoeur, A. Mascarenhas, P. Wei, and F. Schiettekatte, Appl. Phys. Lett., 82, 2245 (2003).

[8] J. Yoshida, T. Kita, O. Wada, and K. Oe, Jpn. J. Appl. Phys. 42, 371 (2003).

[9] K. Oe, Jpn. J. Appl. Phys. 41, 2801 (2002).

[10] K. Alberi, O. D. Dubon, W. Walukiewicz, K. M. Yu, K. Bertulis, and A. Krotkus, Appl. Phys. Lett. 91, 051909 (2007).

[11] M. Usman, C. A. Broderick, A. Lindsay, and E. P. O'Reilly, Phys. Rev. B 84, 245202 (2011).

[12] Z. Batool, K. Hild, T. J. C. Hosea, X. Lu, T. Tiedje, and S. J. Sweeney, J. Appl. Phys. 111, 113108 (2012).

[13] E. C. Young, M. B. Whitwick, T. Tiedje, and D. A. Beaton, Phys. Status Solidi C 4, 1707 (2007).

[14] A. J. Ptak, R. France, D. A. Beaton, K. Alberi, J. Simon, A. Mascarenhas, and C. S. Jiang, J. Cryst. Growth 338, 107 (2012).

[15] H. Fitouri, I. Moussa, A. Rebey, and B. El Jani, J. Cryst. Growth 300, 347 (2007).

[16] R. R. Wixom, L. W. Rieth, and G. B. Stringfellow, J. Cryst. Growth 265, 367 (2004).

[17] D. Fan, Z. Zeng, Xian Hu, V. G. Dorogan, C. Li, M. Benamara, M. E. Hawkridge, Y. I. Mazur, S. Yu, S. R. Johnson, Z. M. Wang, and G. J. Salamo, Appl. Phys.
Lett. 101, 181103 (2012).

[18] A. Janotti, S. H. Wei, and S. B. Zhang, Phys. Rev. B 65, 1152031 (2002).

[19] H. Makholoufi, P. Boonpeng, S. Mazzucato, J. Nicolai, A. Arnoult, T. Hungria, G. Lacoste, C. Gatel, A. Ponchet, H. Carrer, X. Marie, and C. Fontaine, Nanoscale Res. Lett. 9, 123 (2014).

[20] N. Hossain, I. P. Marko, S. R. Jin, K. Hild, S. J. Sweeney, R. B. Lewis, D. A. Beaton, and T. Tiedje, Appl. Phys. Lett. 100, 051105 (2012).

[21] M. Usman, C. Broderick, Z. Batool, K. Hild, T. J. C. Hosea, S. J. Sweeney and E. P. O'Reilly, Phys. Rev. B87, 115104 (2013).

[22] S. V. Morozov, D. I. Kryzhkov, V. I. Gavrilenko, A. N. Yablonsky, D. I. Kuritsyn, D. M. Gaponova, Yu. G. Sadofyev, B. N. Zvonkov, and O. V. Vihrova, Semiconductors 46, 1376 (2012)

[23] S. V. Morozov, D. Kryzhkov, A. N. Yablonskiy, A. V. Antonov, D. I. Kuritsin, D. M. Gaponova, Y. G. Sadofyev, N. Samal, V. I. Gavrilenko, and Z. Krasilnik, J. Appl. Phys. Lett. 113, 163107 (2013).

[24] Y. I. Mazur, V. G. Dorogan, M. Benamara, M. E. Ware, M. Schimdbauer, G. G. Tarasov, S. R. Johnson, X. Lu, S.-Q. Yu, T. Tidje, and G. J. Salamo, J. Phys. D: Appl. Phys. 46, 065306 (2013).

[25] M. K. Shakfa, A. Chernikov, D. Kalincev, S. Chatterjee, X. Lu, R. Johnson, D. A. Beaton, T. Tiedje, and M. Koch, Phys. Status Solidi C 10, 1234 (2013). 